\title{
DASH: A Dynamic 16g Hexapedal Robot
}

\author{
P. Birkmeyer, K. Peterson and R. S. Fearing \\ Department of Electrical Engingeering and Computer Sciences \\ University of California, Berkeley \\ Berkeley, California 94720-1770 \\ Email: \{paulb kevincp ronf\}@eecs.berkeley.edu
}

\begin{abstract}
DASH is a small, lightweight, power autonomous robot capable of running at speeds up to 15 body lengths per second (see video). Drawing inspiration from biomechanics, DASH has a sprawled posture and uses an alternating tripod gait to achieve dynamic open-loop horizontal locomotion. The kinematic design which uses only a single drive motor and allows for a high power density is presented. The design is implemented using a scaled Smart Composite Manufacturing (SCM) process. Evidence is given that DASH runs with a gait that can be characterized using the spring-loaded inverted pendulum (SLIP) model. In addition to being fast, DASH is also well suited to surviving falls from large heights, due to the uniquely compliant nature of its structure.
\end{abstract}

\section{INTRODUCTION}

Highly mobile, small robotic platforms offer several advantages over larger mobile robots. Their smaller size allows them to navigate into more confined environments that larger robots would be unable to enter or traverse such as caves or debris. Small robots are easily transported by either vehicles or humans to be deployed to remote locations as needed. One example includes field workers who need access to otherwise dangerous, inaccessible areas such as collapsed buildings or ones damaged in earthquakes. Small, inexpensive robots are also a key component for rapid installation of ad hoc networks.

As robot size decreases, however, maintaining mobility can become a challenge if climbing means are not available. As objects are larger relative to body dimensions, legs gain advantages over traditional wheels or treads by being able to overcome obstacles greater than hip height. Reduced size also introduces challenges involving controls and power; reduced volume leaves less space for the multiple actuators per leg typically seen on larger robots.

Biology offers a wealth of examples of small creatures with remarkable locomotion abilities, such as the cockroach. Studying these animals has revealed lessons that guide the design of legged robots. One such example is preflexes wherein passive mechanical elements, such as tendons, contribute stabilizing forces faster than the neurological system can respond [1], [2]. Another passive design element that imparts stability is the sprawled alternating tripod gait. In addition to static stability, this limb arrangement has been shown to lend robustness to both fore-aft and lateral perturbations while running [3], suggesting application in legged robots.

The gaits of running animals suggest an additional template useful for stable running. The spring-loaded inverted pendulum (SLIP) model can explain the ground reaction forces during running seen in almost all legged systems with the relative

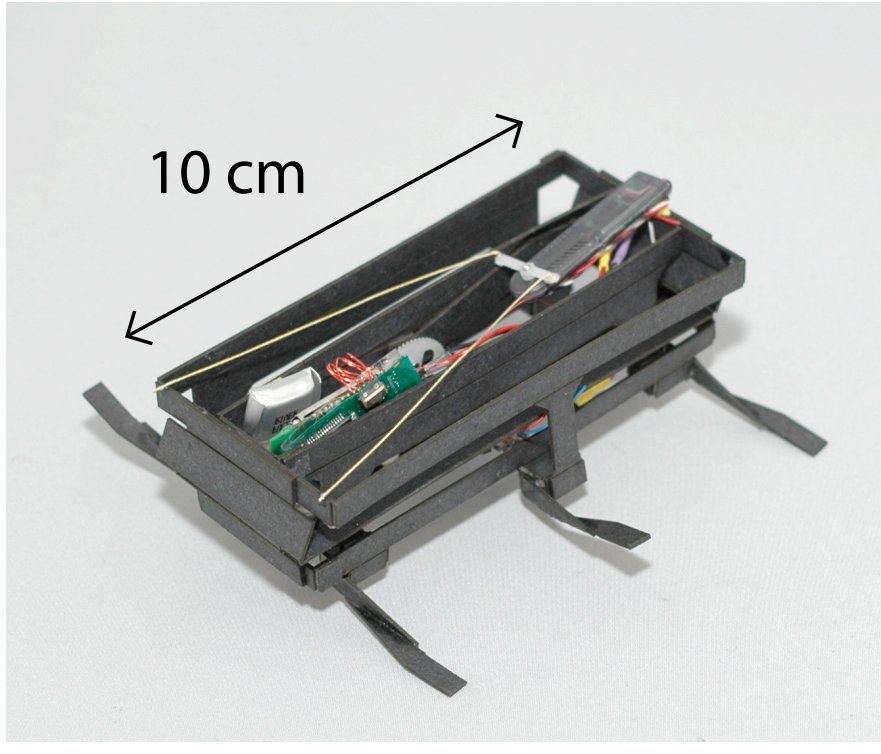

Fig. 1. DASH: a power autonomous hexapedal robot.

stiffness being nearly uniform across all species [4], [5]. The SLIP model has also been shown to impart dynamic stability to horizontal running. Possessing SLIP-like behavior may benefit the mobility of any legged robotic system.

Much work has been done in highly mobile legged robots, some of which incorporate these lessons derived from biology. Examples include Mini-Whegs [6], Sprawlita [7], iSprawl [8], and RHex [9]. These robots incorporate passive compliance to achieve preflex-like behavior and SLIP-like motion. They also all use some form of feed-forward controls, mimicking the central-pattern generator feature from biology [2], [5].

The Dynamic Autonomous Sprawled Hexapod, or DASH, uses design principles from biology and employs a differential drive using only a single motor. It is capable of power autonomous, robust dynamic locomotion at speeds of approximately 15 body-lengths per second $(1.5 \mathrm{~m} / \mathrm{s})$. DASH measures $10 \mathrm{~cm}$ in length, weighs 16.2 grams, and uses wireless communication for feed-forward commands. It is constructed using a scaled Smart Composite Manufacturing (SCM) process that enables fast build times, scalable designs, and lightweight systems [10]. The SCM process also enables falling survivability through an energy absorbing structure and high surface-to-weight ratio. 


\section{Mechanical Design}

The primary design goal of DASH is to achieve highspeed open-loop, feed-forward dynamic running with high power density in a small robotic platform. Neglecting steering actuation, power density can be maximized by using only a single actuator. (Note that with an actuator per leg tripod, on average, half the legs are doing no work.) DC motors, compared to other actuators such as piezoelectric actuators and shape memory alloy wires, provide longer stroke lengths, higher efficiencies, greater power densities, easier electrical interfacing, and operate in a region of frequencies comparable to those observed in insect legged locomotion with appropriate gearing [11].

A key challenge at this size scale is converting the rotary output of a DC motor into useful motions, without the gears and ball bearings which are typically found in each leg of a larger robot. iSprawl uses a crank-slider mechanism attached to push-pull cables to convert the rotation of the motor into directional, linear motions [8], while Mini-Whegs uses a drive-train to transfer the rotational motion directly to the appendages [6]. RHex drives each leg with its own independent motor [9].

Like DASH, both iSprawl and RHex use a hexapedal design with an alternating tripod gait similar to those found often in insects, including the cockroach. Alternating tripods ensure static stability by keeping the center of mass above the support surface formed by at least three legs. DASH, iSprawl, MiniWhegs and SprawlHex (a variation on RHex) [12] all use a sprawled posture. A sprawled hexapedal stance has been shown to encourage robustness to both fore-aft and lateral velocity perturbations [3].

\section{A. Design Overview}

DASH uses stiff linkages and polymer hinge elements to transfer power from the motor to the legs. The mechanism works like an oar, with a circular input trajectory forcing the end of the oar to follow a similar circular output trajectory. In DASH, the DC motor is aligned so that the circular output lies in the sagittal plane. The circular motion of the motor in the sagittal plane is then transferred via the oar mechanism to drive the legs in their own circular orbits. The foot trajectories are biased downward to ensure that the hips, which lie at the bottom of DASH, never contact the ground during locomotion. The design is realized with the SCM process [10], which enable lightweight robots with high power-to-weight ratios, but only allows limited joint rotations due to flexural joints.

\section{B. Hip Design}

The vertical and fore-aft motion of the legs of DASH can be understood separately, and the kinematic models of the hip joint are shown in Figure 2. From the front view, Figure 2(a) shows how the vertical motion of the top beam causes the pair of legs to move in opposite directions vertically. From the top view, Figure 2(b) shows how forward motion of the horizontal beam causes the four-bar linkages to swing one leg forward and swing the opposite leg backwards. These two motions

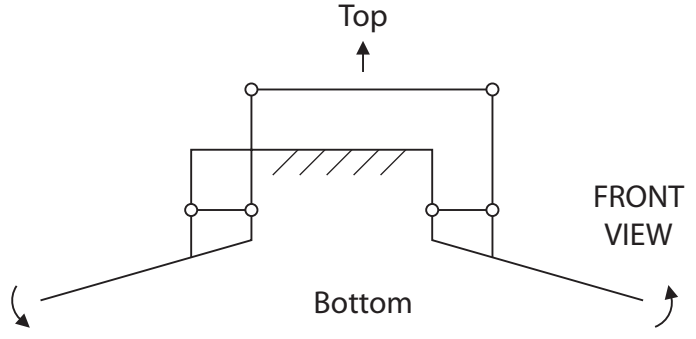

(a)

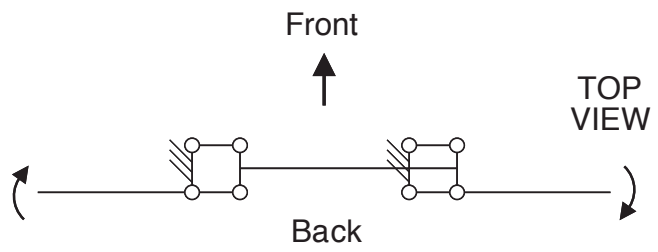

(b)

Fig. 2. Visualization of the degrees of freedom that enable (a) vertical motion and (b) lateral motion of the legs. In both images, the beams to which the hips are attached are grounded.

are then coupled together so that the circular output from the DC motor drives both the vertical displacement required by the model of Figure 2(a) and the horizontal displacement of Figure 2(b). The pair of legs from Figure 2 are repeated three times in the design of DASH to achieve the alternating tripod gait.

The hip joints of DASH in Figure 3 are the realization of the models from Figure 2 in the SCM process. Figure 3 is looking perpendicularly into the sagittal plane of DASH, or along the length of the legs in both Figures 2(a) and 2(b). The circular output from the motor is the dashed circle, and the motor progressively rotates while moving from Figure 3(a) to Figure 3(d). The black vertical beams transfer the force from the motor to the hip joints. Vertical motion of the black beams creates vertical motion of the leg as in Figure 2(a), and horizontal motion of the beams creates horizontal motion the leg as in Figure 2(b). Note how the two adjacent legs in Figure 3 are 180 degrees out of phase, as is needed for the alternating tripod gait. These hip joints are then repeated three times to get six legs which move with the proper phase.

\section{Differential Drive}

The hip design in Fig. 3 is part of a differential mechanism that relies on the input moving in a circle relative to the fixed beam to which the hips are attached. The mechanism which achieves this differential drive motion is shown in Figure 4. The light, transparent beams on the bottom have the dark circular motor rigidly mounted to them, and the output of the motor is rigidly attached to the dark beams on the top. The five thin linkages connecting the bottom transparent horizontal beams to the top dark horizontal beams enforce a constraint which keeps the beams parallel with the horizontal plane. They form two parallel four-bar mechanisms with a shared horizontal beam that runs between the dark and transparent 


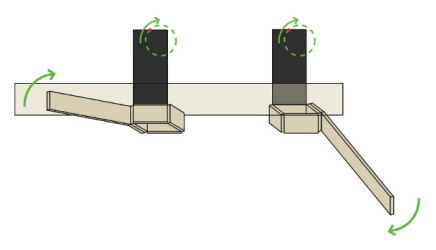

(a)

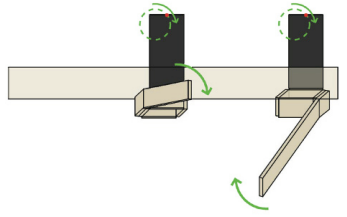

(b)

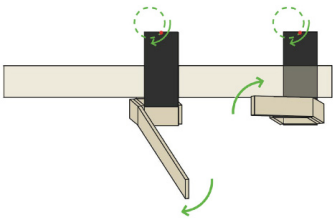

(c)

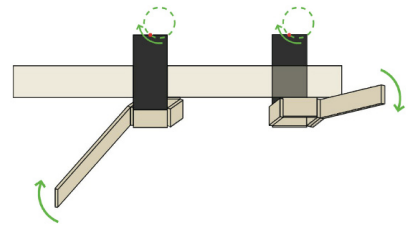

(d)

Fig. 3. Four different positions of sample hip joints from DASH presented from the side of DASH. The black beams are attached to a circular input. The beams move in the dotted paths, causing the motion of the legs. The axes of rotation for the hips lie on opposite sides relative to their respective inputs, creating an antiphase motion. Moving from left to right, both the circular input and the legs move clockwise.

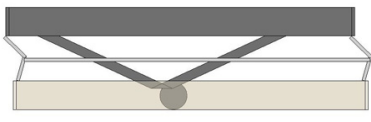

(a)

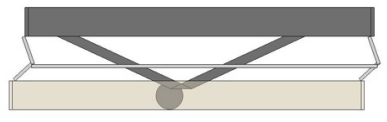

(b)

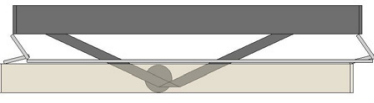

(c)

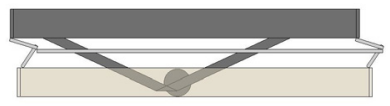

(d)

Fig. 4. Looking from the side of DASH, linkages connect the top beams with the bottom beams and force them to remain parallel. The linkages form two parallel four-bar mechanisms that share the horizontal beam that runs between the bottom and top beams. The round motor is mounted rigidly to the bottom beams, which are transparent in order to reveal the motor. The motor output is rigidly connected to all of the dark beams. The parallel constraint means that every point on the top beams move in the same circular path as the motor output, keeping the motion of the legs in each tripod identical. Note that the positions correspond with the position of the input in Figure 3.

beams. It is important to note that the bottom light horizontal beams in Figure 4 are the same as those in Figure 2; the hip joints have been removed from Figure 4 for simplification. Also the dark vertical beams of Fig. 3 are attached to underside of the horizontal dark beams of Fig. 4. The parallel constraint thus forces all points on the dark beams to travel in the same circular path as the output of the motor, which in turn means that each hip joint goes through the same angular displacements. This differential drive mechanism has a floating ground where only the relative motion of the bottom and the top of the robot achieve useful output at the legs. This differs from other robots such as iSprawl and Mini-Whegs wherein the entire drive train is grounded to the same structure to which the hips are attached.

\section{Leg Design}

Two different leg designs are used with DASH. The first is a stiff, more horizontal design (Fig. 5(a)) and the second is an angled design with built in compliance at the foot (Fig. 5(b)). The compliant leg has one flexure joint that is free to rotate when pressed into the ground. The rigidity of the joint determines the compliance of the leg.

\section{E. Body Design and Steering}

The cardboard beams used to construct DASH are rigid when forces are directed along the face of the beam; however, because the beams are only approximately 900 microns thick, they lack rigidity when subjected to forces directed into the face of the beam. All the beams in DASH are oriented to present the most stiffness in both the fore-aft and vertical axes, since these are the two directions which experience the greatest forces during locomotion. Stiffness in these directions is required for good power transmission to the legs. There are instances, however, when loading occurs in the weak axes of

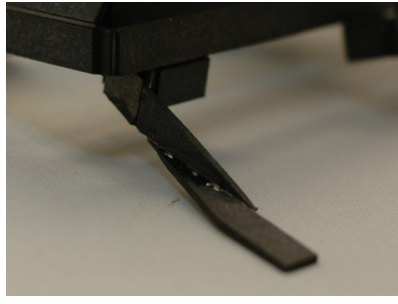

(a)

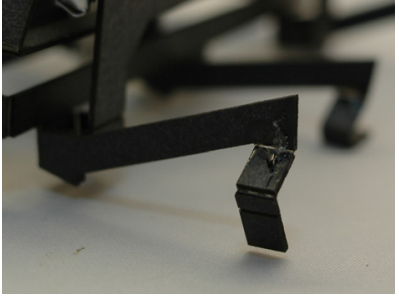

(b)
Fig. 5. Two different leg designs used by DASH. (a) is the stiff, horizontal design and (b) is the compliant design.

the beams. The lack of off-axis rigidity introduces compliance in the structure as the beams deform under loads. This is in addition to the small amounts of compliance already present due to polymer hinges not perfectly emulating pin joints.

During straight-ahead running, the arc swept out by the legs' fore-aft motion is centered about a midpoint perpendicular to the body, as in Figure 2(b). These leg motions are balanced on both sides of the body and drive the robot straight. To steer, skewing the frame of DASH biases the midpoint of the arcs swept by the legs as shown in Figure 6. This shifts location and direction of the ground reaction forces, imparting a moment on the body. A one gram shape-memory alloy SmartServo RC-1 from TOKI Corporation, mounted to the rear of DASH, pulls on the front corners of DASH's frame, resulting in a skewed frame and an induced turn. The direction of the turn is dependent upon which corner of DASH is pulled toward the SmartServo. No control input to the SmartServo results in straight-ahead motion. 


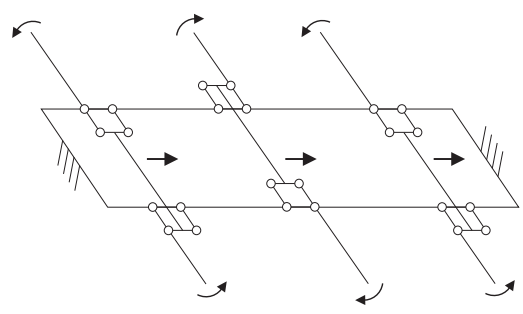

Fig. 6. A model of how the kinematics change when the body is contorted. The midpoint of the fore-aft strokes are biased forward on one side of the body and backwards on the opposite side. This induces a moment on the body and results in turning. See companion video.

\section{RESULTS}

DASH is capable of running at 15 body-lengths per second (1.5 meters per second). This speed was observed on a DASH with no steering actuator mounted, but the addition of this actuator appears to have little effect on the top speed. The entire system weighs only 16.2 grams with battery and electronics. Its body is $10 \mathrm{~cm}$ long and $5 \mathrm{~cm}$ wide. Including legs, it measures approximately $10 \mathrm{~cm}$ wide and $5 \mathrm{~cm}$ tall. The electronics package includes a microcontroller, motor driver, and Bluetooth communication module for wireless operation [13]. Running at full power with no steering control, its $3.7 \mathrm{~V}$ $50 \mathrm{mAh}$ lithium polymer battery provides approximately 40 minutes of battery life.

\section{A. Running Performance}

Figure 7 shows DASH running at 11 body-lengths per second. From the sequence of images, the alternating tripod gait produced by the structure can be seen. In general, there are aerial phases between the touchdown of the alternating tripods with aerial phases taking up to 30 percent of a full cycle.

When DASH runs, the center of mass follows a roughly sinusoidal trajectory, which is indicative of the SLIP model. Fig. 8 shows the trajectory during another example of horizontal running. The center of mass is tracked throughout the run, and it follows a stable sinusoidal motion. This trajectory of the center of mass is similar to those seen in running animals [4], [5].

The SLIP model of ground reaction forces is shown in Fig. 9(a). In the model, when a system makes initial contact with the ground, it decelerates as it stores energy in the system. As the normal force peaks, however, the fore-aft force becomes positive as the energy is returned to the system and it accelerates forward. Analysis of the SLIP model shows that these ground reaction forces lend dynamic stability when running in the horizontal plane [5]. The ground reaction forces of a cockroach are shown in Fig. 9(b). The cockroach data records a cockroach running in an alternating tripod gait where the animal never undergoes an aerial phase, causing the normal force to never reach zero. DASH was tested on a force platform to see if it exhibits similar ground reaction forces to those of natural systems, including the cockroach. A representative

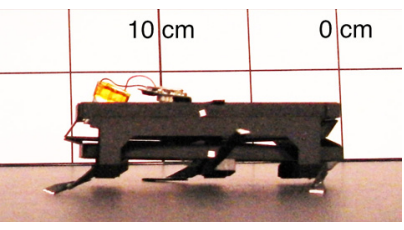

(a) $\mathrm{t}=0 \mathrm{~ms}$

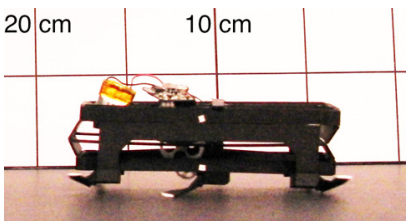

(c) $\mathrm{t}=33.3 \mathrm{~ms}$

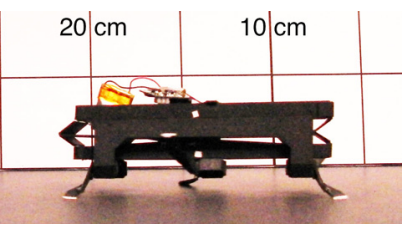

(e) $\mathrm{t}=66.7 \mathrm{~ms}$

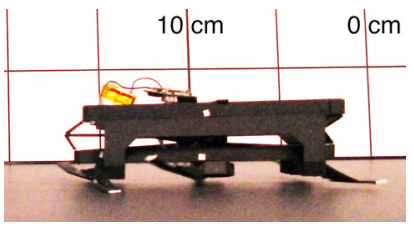

(b) $\mathrm{t}=16.7 \mathrm{~ms}$

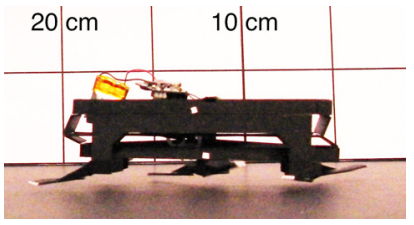

(d) $\mathrm{t}=50 \mathrm{~ms}$

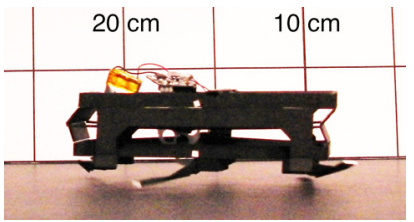

(f) $\mathrm{t}=83.3 \mathrm{~ms}$
Fig. 7. DASH exhibits dynamic horizontal locomotion. (a) depicts the beginning of one tripod stance and (b) shows the end of the tripod stance. (c) captures the middle of the second tripod stance. (e) shows the middle of the first tripod stance. (d) and (f) both show aerial phases. The ends of the legs are painted white for better visibility.

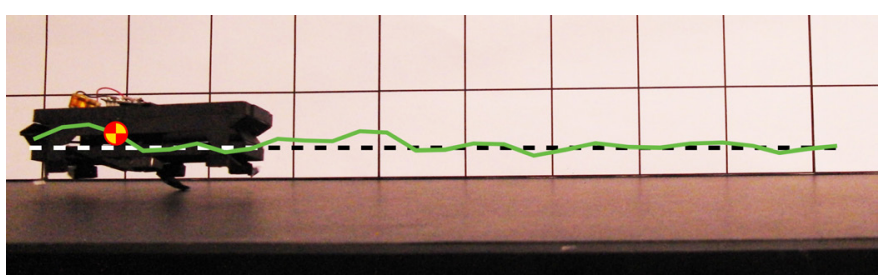

Fig. 8. Center of mass tracked over nearly one half second. The center of mass follows an approximate sinusoidal trajectory. The grid pattern marks 5 $\mathrm{cm}$ increments. The dashed line shows the resting position of the center of mass.

sample of filtered data showing DASH running on a horizontal force platform is in Fig. 9(c). Each large increase in normal force corresponds with a single tripod making contact with the ground. Just as in the SLIP model and with the cockroach, there is a deceleration upon initial contact followed by an acceleration. The phase relationship between the fore-aft and normal forces are the same as both the model and what is observed in the cockroaches. The shapes of the force curves are also very similar, with some slight differences between the measured forces and the sinusoidal forces of the SLIP model.

When fitting different animals to the SLIP model, the stiffness of the equivalent spring in the model has been found to be remarkably similar for many different animals, when normalized by mass, leg length, and leg number. The relative stiffness of all of the legs is $k_{\text {rel }}=\left(F_{\text {vert }} / \mathrm{mg}\right) /(\Delta l / l)$ where $F_{\text {vert }}$ is the vertical ground reaction force measured at midstance, $\Delta l$ is the compression of the center of mass, $l$ is the average height of the center of mass, and $m g$ represents 


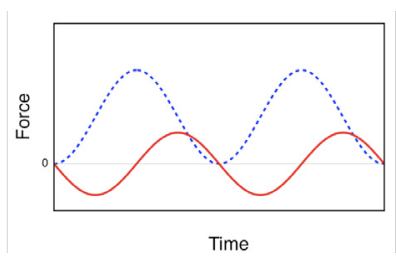

(a)

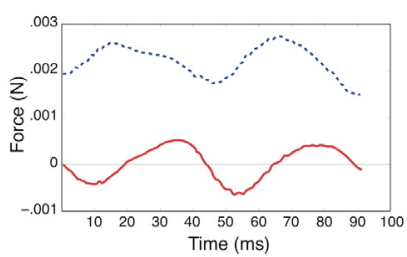

(b)

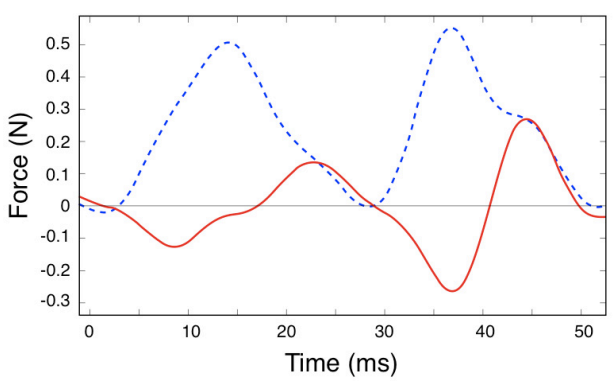

(c)

Fig. 9. Dynamic locomotion in the horizontal plane has been shown to be similar across many legged systems as modeled by SLIP. In the figures, red solid lines indicate fore-aft forces and blue dotted lines indicate normal forces into the ground. Negative fore-aft forces connote decelerating forces and positive fore-aft forces connote accelerating forces. Positive normal forces indicate forces pushing down on the surface. The SLIP model is shown in (a), the ground reaction forces of a cockroach [14] are shown in (b), and the ground reaction forces of DASH are shown in (c).

weight [15]. The relative stiffness of an individual leg $k_{r e l, i n d}$ is found by dividing the relative stiffness by the number of legs that support the body during the stance phase. For DASH, $k_{r e l, i n d}=9$ with the horizontal leg design and $k_{r e l, i n d}=11.3$ with the compliant leg design. As shown in Fig. 10, the relative stiffness of DASH fits the trend found in animals during dynamic locomotion. The relative stiffness of DASH is very similar for both of the current leg designs, despite each design having different individual stiffness properties. This is due to the serial compliance that exists in the hips and throughout the body of DASH.

Because of the structure of DASH, the overall stiffness depends on both the body and the leg design. To measure the overall stiffness, the motor was locked in place as the

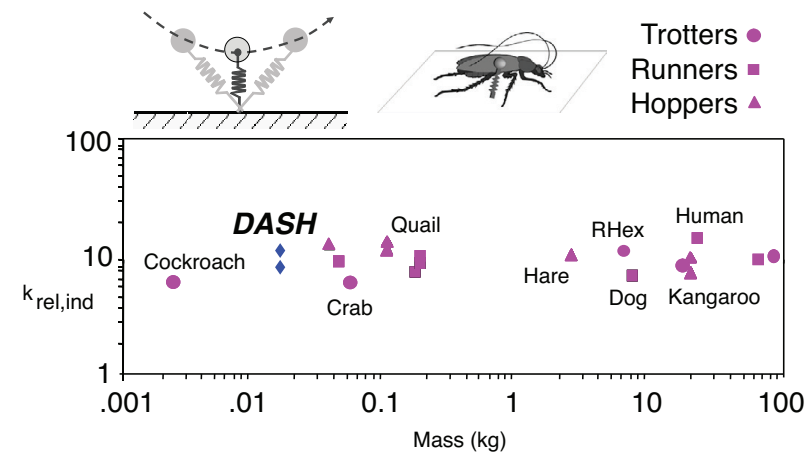

Fig. 10. Relative stiffness of individual legs vs. mass of various animals and DASH with 2 leg designs. Reprinted from [5] with permission of Full.

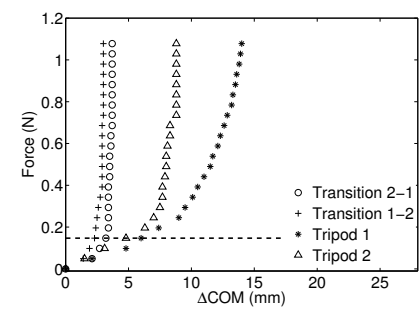

(a)

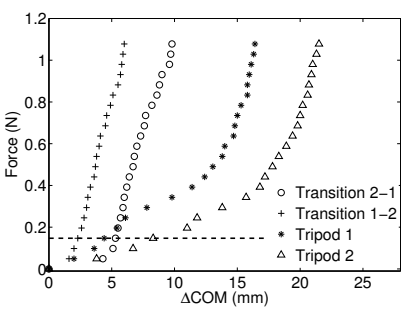

(b)
Fig. 11. Stiffness of DASH in the normal direction for different leg types and stances. The basic horizontal leg design is shown in (a), and the compliant design is shown in (b). The stiffness was measured for both tripods, as well as with 6 legs on the ground between each tripod phase. The horizontal dashed line represents the weight of the robot.

phase within the stride of DASH will affect the stiffness. The measurement was performed by adding mass in small increments to the top of the robot and measuring the displacement of the center of mass. Fig. 11 shows the stiffness curves for both the basic horizontal leg design (Figure. 5(a)) and the more compliant design (Figure 5(b)). For both leg designs, the curves have 2 distinct shapes corresponding to DASH having 3 legs or 6 legs on the ground. When only 3 legs are in contact, the body is able to flex significantly and we obtain a low stiffness, while stiffness is higher when all 6 legs are in contact with the ground. The stiffness curve has two regions. In the first region, the stiffness is low $(30 \mathrm{~N} / \mathrm{m}$ for horizontal legs and $22 \mathrm{~N} / \mathrm{m}$ for compliant legs). However, as the normal load on the robot is increased, the serial compliance in the structure allows the body to deform until all 6 legs make contact with the ground. At this point the stiffness of the robot changes, and exhibits a comparable stiffness $(240 \mathrm{~N} / \mathrm{m})$ to the case with the robot starting with 6 legs on the ground.

\section{B. Turning Results}

DASH demonstrates that turning can be achieved with relatively simple modifications of kinematics. The top speed of DASH is negligibly affected by the turning actuator, but turning is most successful when operating at a 20 percent duty cycle applied to the main DC motor. Figure 12 shows a turning maneuver of DASH, first turning left and then immediately turning right. DASH turns approximately 50 degrees per second to left and 55 degrees per second to the right when applying a maximum turning input and a 20 percent duty cycle to the main drive.

\section{Step Climbing}

DASH is able to mount obstacles greater than its own body height using only feed-forward controls. The compliance throughout the structure can compensate for the differences in foot position when running on uneven terrain, and power is still successfully delivered to the feet. Though lacking control of individual foot placement, DASH is eventually able clear the $5.5 \mathrm{~cm}$ obstacle in Figure 13. This is near the upper limit of step heights DASH can surmount. 


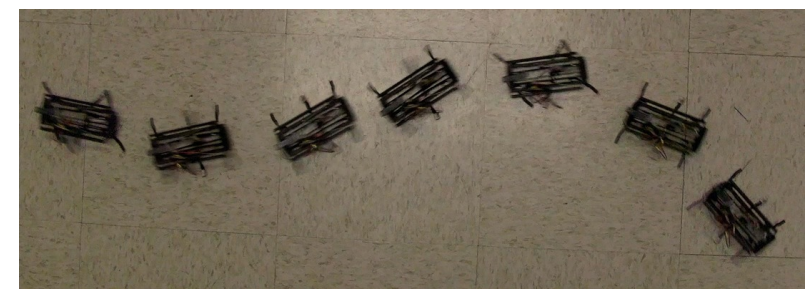

Fig. 12. Controlled turning maneuvers from companion video using a single turning actuator

\section{Surviving Falls}

Several tests were carried out to show the capability of DASH to survive falls from large heights (see video). After multiple drops of 7.5 meters, 12 meters, and 28 meters (75, 120 and 280 body lengths) on to concrete, the robot remained operational, with no damage. From 28 meters, the velocity at impact is approximately $10.3 \mathrm{~m} / \mathrm{s}$. This has been verified to be the terminal velocity using wind tunnel experiments, indicating it can survive falls from any height. Despite this high impact velocity, the impact energy is only $0.795 \mathrm{~J}$ due to the lightweight construction. As can be seen from Fig. 14, the body contorts dramatically upon impact, in the process absorbing a significant fraction of impact energy. In these images, the body was falling at approximately 6.5 meters per second just prior to impact.

\section{Discussion of Results AND CONCLUSIONS}

With a top speed of approximately 15 body-lengths a second, DASH meets the goal of achieving high-speed running using a single drive actuator. It does so using a novel differential drive and the circular output of a DC motor to create two antiphase circular leg motions. The resulting kinematics create an alternating tripod gait that exhibits dynamic behavior and mimics the ground reaction forces seen in natural systems. Turning is achieved using a simple modification to the kinematics. It is constructed using a scaled SCM process which uses bi-rigid beams and polymer hinges to construct a very lightweight system.
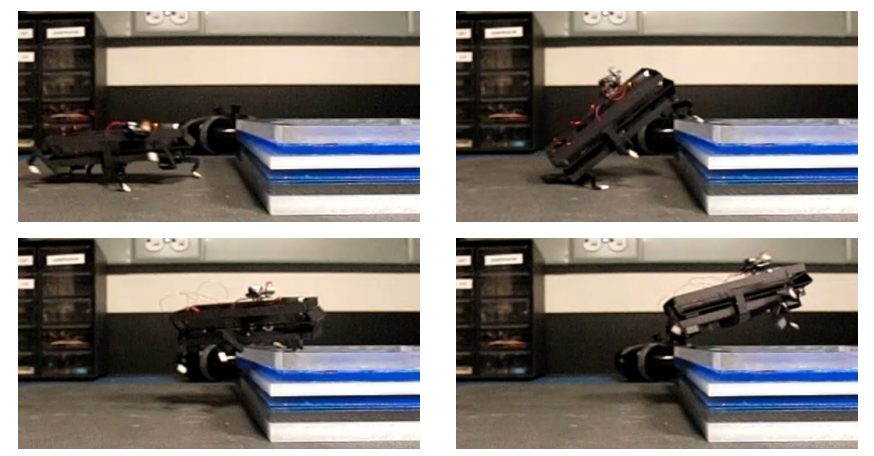

Fig. 13. Images from companion video of DASH climbing over a stack of acrylic. The obstacle is approximately $5.5 \mathrm{~cm}$ tall.

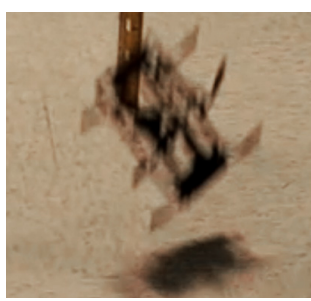

(a) $\mathrm{t}=0 \mathrm{~ms}$

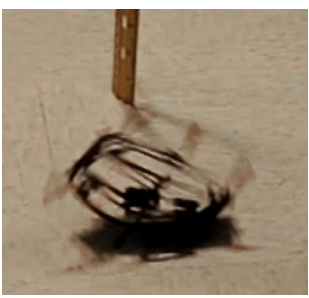

(c) $\mathrm{t}=16.7 \mathrm{~ms}$

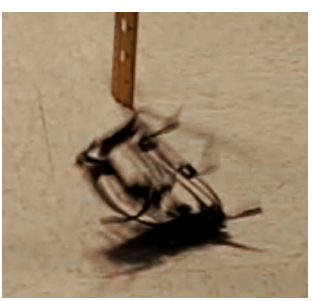

(b) $\mathrm{t}=10 \mathrm{~ms}$

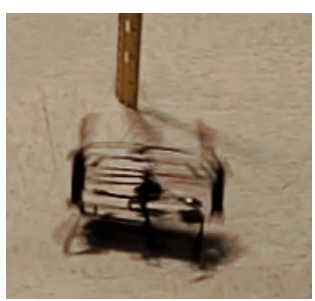

(d) $\mathrm{t}=23.3 \mathrm{~ms}$
Fig. 14. Images from companion video of DASH impacting the ground at a velocity of approximately $6.5 \mathrm{~m} / \mathrm{s}$. (b) and (c) show the great contortion that the body undergoes during a high velocity impact. By (d), the body has almost fully recovered its original shape. The compliance of the structure enables it to withstand high impact velocities and survive without damage.

When running, the lower stiffness value seen for 3 legged stance contributes positively to horizontal locomotion in several ways. If it were perfectly rigid, the gait would be much more unforgiving and less smooth. Because of the compliance present in the structure, a more SLIP-like gait can be achieved, improving the robot's overall locomotion.

DASH compares favorably to other small legged robots in terms of running speed in body lengths per second (Table I). At 15 body lengths per second, DASH is faster than both Mini-Whegs and RHex, and is comparable to iSprawl. Its dynamic locomotion exhibits SLIP behavior similar to that of natural systems. In addition to being fast, DASH also offers several unique characteristics. While DASH is not significantly different in volume from Mini-Whegs, it is approximately 10 times lighter. This is a consequence of the SCM process which replaces traditional solid structural elements with hollow, folded structures. The light weight enables a battery life of 40 minutes at top speed with only a 1.8 gram $50 \mathrm{mAh}$ battery. Upgrading to a larger battery, such as a 2.6 gram $90 \mathrm{mAh}$ battery, could dramatically increase battery life while having a minimal impact on locomotion speed.

The scaled SCM process, in addition to providing a low density robot, provides a mechanical structure which can survive falls from large heights. There are several factors that influence the survival rate: velocity at impact, surface compliance, number and compliance of limbs, orientation, and impact load per unit area [17]. The design of DASH provides many of the characteristics necessary for surviving falls. Light weight, low density robots have a decided advantage in falling, because of their reduced velocity, and low impact energy when hitting the ground. Because of the distributed compliance throughout the body of DASH, the robot is capable of surviving falls 
TABLE I

SELECT PROPERTIES OF COMPARABLE ROBOTS ${ }^{1}$

\begin{tabular}{|c|c|c|c|c|}
\hline & DASH & iSprawl [8] & Mini-Whegs [6] & RHex [9], [16] \\
\hline Size $(\mathrm{cm})$ & $10 \times 5 \times 10$ & $15.5 \times 11.6 \times 7$ & $9 \times 6.8 \times 7.2$ & $53 \times 20 \times 15$ \\
\hline Mass $(\mathrm{g})$ & 16.2 & 300 & 146 & 7500 \\
\hline Speed (body-lengths/second) & 15 & 15 & 10 & 5 \\
\hline Leg Frequency $(\mathrm{Hz})$ & 17 & 14 & 22 & 5 \\
\hline Density $(\mathrm{g} / \mathrm{cc})$ & 0.03 & 0.24 & 0.33 & 0.48 \\
\hline Battery life $(\mathrm{min})$ & 40 & 5 & N/A & 30 \\
\hline Max Power Density ${ }^{2}(\mathrm{~W} / \mathrm{kg})$ & 20 & 8.6 & 8.2 & 8 \\
\hline Cost of Transport $(\mathrm{J} / \mathrm{kg} / \mathrm{m})$ & 14.7 & 17.4 & 8.9 & 20 \\
\hline
\end{tabular}

${ }^{1}$ Several values in this table were not explicitly published in the literature. They were calculated to the best of our ability using the available data.

${ }^{2}$ The power density requires knowing the force delivered to the ground during locomotion. An upper bound for this value is calcutated for iSprawl, Mini-Whegs, and Rhex using the rated output power for the motors. The value for DASH was calculated using the experimental output power of the motor.

regardless of the landing orientation or compliance specifically in the limbs. The cardboard structure of the robot functions as a roll cage around the more fragile parts of the robot: the motor, control board and battery. MiniWhegs has survived falls of 0.9 meters [6] without damage as did RHex from a 6 meter height [18]. In general, however, there are very few claims of robustness to falling in legged robots. Since DASH survives high falls without damage and is low cost, it can take risks with falls which another robot might not survive.

Future work will explore the dynamics of turning in DASH and consider alternative turning strategies, such as varying kinematics or leg stiffnesses to achieve turning. Using the measured stiffness of the leg tripods, it is estimated that the resonant frequency of DASH is around $8 \mathrm{~Hz}$, roughly half of the frequency used to achieve the highest velocities. It is possible that bringing the resonant frequency closer to the operating frequency would benefit locomotion and efficiency. Some combination of reducing mass by switching materials from cardboard to fiberglass or carbon fiber and increasing the stiffness of the legs and drive train could bring the resonant frequency closer to the operating frequency.

The space of leg designs also needs to be explored further. Different leg designs could also increase the stability and speed of DASH. Other models derived from nature, such as the Lateral Leg Stiffness model, have been shown to contribute to stability and can also allow for rapid maneuvers [19]. Other leg designs might help to bring DASH more in line with these models. Alternate legs could also enable testing and optimization over different terrains and media, including random rough terrain or sand.

\section{ACKNOWLEDGMENT}

The authors thank Alexis Birkmeyer for her help with the figures. Thanks also to the Center for Integrative Biomechanics in Education and Research for the use of their force platform. This work is supported by the NSF Center of Integrated Nanomechanical Systems and the United States Army Research Laboratory under the Micro Autonomous Science and Technology Collaborative Technology Alliance.

\section{REFERENCES}

[1] I. E. Brown and G. E. Loeb, "A reductionist approach to creating and using neuromusculoskeletal models," in Biomechanics and neural control of posture and movement, J. M. Winters and P. E. Crago, Eds. New York: Springer, 2000.

[2] S. Sponberg and R. J. Full, "Neuromechanical response of musculoskeletal structures in cockroaches during rapid running on rough terrain." J Exp Biol, vol. 211, no. 3, pp. 433-446, Feb 2008.

[3] T. M. Kubow and R. J. Full, "The role of the mechanical system in control: a hypothesis of self-stabilization in hexapedal runners," Phil. Trans. R. Soc., vol. 354, pp. 849-851, 1999.

[4] R. Blickhan and R. J. Full, "Similarity in multilegged locomotion: Bouncing like a monopode," Journal of Comparative Physiology A: Neuroethology, Sensory, Neural, and Behavioral Physiology, vol. 173, no. 5, pp. 509-517, 111993.

[5] P. Holmes, R. J. Full, D. Koditschek, and J. Guckenheimer, "The dynamics of legged locomotion: Models, analyses, and challenges," SIAM Review, vol. 48, no. 2, pp. 207-304, 2006.

[6] J. M. Morrey, B. Lambrecht, A. D. Horchler, R. E. Ritzmann, and R. D. Quinn, "Highly mobile and robust small quadruped robot," in Proceedings of the 2003 IEEE/RSJ International Conference on Intelligent Robots and Systems, Las Vegas, NV, 2003, pp. 82-87.

[7] J. G. Cham, S. A. Bailey, J. E. Clark, R. J. Full, and M. R. Cutkosky, "Fast and robust: Hexapedal robots via shape deposition manufacturing," International Journal of Robotics Research, vol. 21, no. 10, 2002.

[8] S. Kim, J. E. Clark, and M. R. Cutkosky, "isprawl: Design and tuning for high-speed autonomous open-loop running," Int. J. Rob. Res., vol. 25, no. 9, pp. 903-912, 2006.

[9] U. Saranli, M. Buehler, and D. E. Koditschek, "Rhex: A simple and highly mobile hexapod robot," International Journal of Robotics Research, vol. 20, no. 7, pp. 616 - 631, July 2001.

[10] A. Hoover and R. Fearing, "Fast scale prototyping for folded millirobots," IEEE International Conference on Robotics and Automation, pp. 886-892, May 2008.

[11] E. E. Steltz, "Redesign of the micromechanical flying insect in a power density context," Ph.D. dissertation, EECS Department, University of California, Berkeley, May 2008.

[12] H. Komsuoglu, K. Sohn, R. J. Full, and D. E. Koditschek, "A physical model for dynamical arthropod running on level ground," ScholarlyCommonsPenn, Tech. Rep., 2008.

[13] F. G. Bermudez and R. Fearing, "Optical flow on a flapping wing robot," in IROS, 2009.

[14] R. Full and M. Tu, "Mechanics of six-legged runners," J Exp Biol, vol. 148, no. 1, pp. 129-146, 1990.

[15] D. E. Koditschek, R. J. Full, and M. Buehler, "Mechanical aspects of legged locomotion control," Arthropod Structure and Development, vol. 33, no. 3, pp. $251-272,2004$.

[16] D. Campbell, "Bounding and stair descent in the hexapod rhex," Master's thesis, McGill University, February 2004.

[17] J. M. Cameron and R. C. Arkin, "Survival of falling robots," W. J. Wolfe and W. H. Chun, Eds., vol. 1613, no. 1. SPIE, 1992, pp. 91-102.

[18] E. Z. Moore, "Leg design and stair climbing control for the rhex robotic hexapod," Master's thesis, McGill University, January 2002.

[19] J. E. Seipel, P. J. Holmes, and R. J. Full, "Dynamics and stability of insect locomotion: a hexapedal model for horizontal plane motions," Biol. Cybern., vol. 91, no. 2, pp. 76-90, 2004. 